\title{
Electromagnetic Induction in the Earth by an Aperiodic Non-Uniform Current Source
}

\author{
R.D. Hibis, JR. and F.W. Jones \\ Department of Physics and the Institute of Earth and \\ Planetary Physics, University of Alberta, \\ Edmonton, Alberta, Canada
}

(Received January 29, 1973; Revised October 21, 1973)

\begin{abstract}
Electromagnetic induction in a two-dimensional earth by a non-uniform, spatially varying, aperiodic current source is considered. The two-dimensional diffusion equation (for the $E$-polarization case) is solved by a separation of variables and is related to the current source by a Fourier integral which synthesizes the actual fields from elementary fields with time dependence $\exp (i \omega t)$ over a horizontally stratified earth. An aperiodic source is obtained by summing the single frequency solutions in a Fourier transformation. The spatial variation is achieved by superimposing the electromagnetic fields of separate aperiodic, non-uniform current sources which vary independently with time. Variations of the magnetic field components with time at the surface of the earth are studied.
\end{abstract}

\section{Introduction}

Little attention has been given to the problem of electromagnetic induction in the earth by a non-uniform source which is temporally aperiodic and also exhibits spatial variation with time. PRICE (1950) examined aperiodic inducing fields and considered the efiects due to a sudden increase in the inducing field and he indicated how the solution for any other time variation could be obtained. The special case of a stationary line current parallel to the surface of the earth in which a sudden increase in the line current intensity took place was considered by him. Also, WaIt (1954) considered the transient response of the tangential electric field for a unit step increase in the magnetic field. The calculations by WAIT (1954) which are applicable to non-uniform sources, were made for subsurface layers of different conductivity and susceptibility.

Hermance and Peltier (1970) considered the magnetic fields of a line current, and Peltier and Hermance (1971) of a Gaussian electrojet. Hibbs and Jones (1973a,b) have considered symmetric and non-symmetric source distributions over both layered and laterally non-uniform conductors.

KISABETH and Rostoker (1971) show that the fields associated with polar magnetic substorms are aperiodic and indicate that the structure of polar magnetic substorms is controlled by the intensification of the currents at the northern 
and/or southern borders of the auroral electrojet. They propose that quasi-periodic intensifications of the northern border of the electrojet are responsible for the jagged structure noted on magnetograms during the substorms. It appears that multi-current systems exist which are responsible for the field fluctuations associated with polar magnetic substorms. It is therefore of interest to consider the effects at the surface of a finitely conducting earth due to multiple current system distributions.

\section{The Two-Dimensional Double Current System}

Within the limitations of the two-dimensional problem a double current system may be studied by an adaptation of the method which PrICE (1950) originally described, and Peltier and Hermance (1971) and Hibbs and Jones $(1973 \mathrm{a}, \mathrm{b})$ have used for investigating non-uniform sources. The two current systems, which may be spatially displaced relative to one another and which may be made to vary aperiodically with time, can have maximum intensities which may occur at different times and so the current system as a whole will vary spatially.

In the present discussion, two current sheets with different spatial distributions of intensity flow above an earth with a horizontally layered conductivity distribution. One system has a Gaussian current intensity distribution, $\exp \left(-y^{2} / 2 \kappa^{2}\right)$. The other system has a current intensity distribution $y \cdot u(y) \cdot \exp (-a y)$ where $u(y)$ is the unit step function. Each system may vary aperiodically with time by summing the solutions for different frequencies through the use of the Fourier transform. The spatially varing solution is obtained from the superposition of the separate aperiodic time solutions.

\section{Method of Solution}

The coordinate system is shown in Fig. 1. The Gaussian current source is

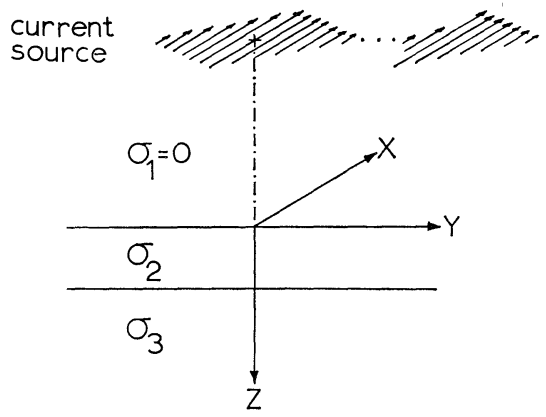

Fig. 1. The co-ordinate system, symmetric and non-symmetric source configuration and layered earth structure. 


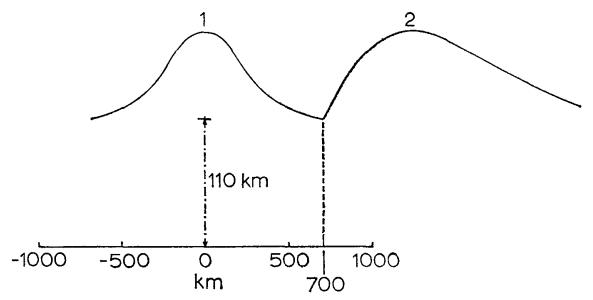

Fig. 2. Source position with respect to region where magnetic field component values are calculated. The curves (1) and (2) represent the current intensity distributions. The currents are sheet currents flowing parallel to the earth at height $110 \mathrm{~km}$.

symmetric about the origin (see Fig. 2) and the current flows parallel to the surface of the earth at a height of $110 \mathrm{~km}$. In the following, this source will be referred to as the symmetric source. The second source, with current intensity distribution of the form $y \cdot u(y) \cdot \exp (-a y)$ will be referred to as the non-symmetric source. The current associated with this latter source also flows parallel to the surface of the earth at a height of $110 \mathrm{~km}$. The non-symmetric source has been displaced $700 \mathrm{~km}$ in the positive $y$ direction from the symmetric source (see Fig. 2). Values for the magnetic field components at the surface of the earth due to the two current systems are obtained at intervals of $100 \mathrm{~km}$ in the region $-1000 \mathrm{~km} \leqq y \leqq 1000 \mathrm{~km}$.

For the $E$-polarization case, when the period is sufficiently long so that displacement currents may be neglected, the equation to be solved is given by:

$$
\frac{\partial^{2} E_{x}}{\partial y^{2}}+\frac{\partial^{2} E_{x}}{\partial z^{2}}=i \omega \mu_{0} \sigma(z) E_{x} \quad \text { (JoNes and PRICE, 1970) }
$$

where $\mu_{0}, \sigma(z)$ and $\omega$ are the permeability of free space, the conductivity of the medium which can vary with $z$, and the angular frequency respectively. The corresponding equation is written in e.m.u. by Jones and Price, but MKS units are used throughout the present work. Certain symmetric and non-symmetric current distributions can be used, and solutions for this equation for the different source distributions may be obtained.

Peltier and Hermance (1971) show that for a horizontally layered earth and for an inducing field with sinusoidal time dependence, the solution of Eq. (1) which describes the elementary electric field in the $n$th layer can be written

$$
\left.E_{x}^{n}=\left\{A_{n}(\theta) \exp \left(-\theta_{n} z\right)+B_{n}(\theta) \exp \left(\theta_{n} z\right)\right\} V(s) \cos (s y)+W(s) \sin (s y)\right\}
$$

where $\theta_{n}^{2}=s^{2}+i \omega \mu_{0} \sigma_{n}$, and $A_{n}(\theta)$ and $B_{n}(\theta)$ are constants which depend upon the conductivity configuration.

The quantity $s$ is the spatial wave number, and $\sigma_{n}$ is the conductivity of the $n$th layer. 
For a symmetric source over a two-layered earth, Peltier and Hermance (1971) showed that

$$
V(s)=-\frac{i I_{0} \omega \mu_{0} \kappa}{\sqrt{2 \pi} s} \exp \left(s z_{0}-s^{2} \kappa^{2} / 2\right) \quad \text { and } \quad W(s)=0
$$

where $I_{0}$ and $Z_{0}$ are the maximum current intensity, and height of the source respectively, and $\kappa$ is the standard deviation at the source. Also, HrbBs and JoNes (1973 b) showed for a non-symmetric current source of distribution

$$
\left.I_{x}(y)=I_{0} \cdot(y-b) \exp \{-a(y-b)\} u(y-b)\right\}
$$

where $b$ and $a$ are constants and $u(y-b)$ is the unit step function, that

where

$$
\begin{aligned}
& V(s)=c^{\prime}(s) \cdot \frac{\left(a^{2}-s^{2}\right) \cos (s b)-2 a s \sin (s b)}{\left(a^{2}+s^{2}\right)^{2}}, \\
& W(s)=c^{\prime}(s) \cdot \frac{2 a s \cos (s b)+\left(a^{2}-s^{2}\right) \sin (s b)}{\left(a^{2}+s^{2}\right)^{2}}
\end{aligned}
$$

$$
c^{\prime}(s)=-\frac{i \omega \mu_{0} I_{0}}{2 \pi s} \exp \left(z_{0} s\right)
$$

Integrating the eigenfunction for $E_{x}^{n}$ given above over all values of $s$, the electric field can be found at any point where its value is desired. That is

$$
\begin{aligned}
E_{x}^{n}(y, z)= & \int_{0}^{\infty}\left\{A_{n}(\theta) \exp \left(-\theta_{n} z\right)+B_{n}(\theta) \exp \left(\theta_{n} z\right)\right\} \\
& \times\{V(s) \cos (s y)+W(s) \sin (s y)\} \mathrm{d} s .
\end{aligned}
$$

The half-space above the earth is taken as the first layer and if $A_{1}(\theta)=1$ then $V(s)$ and $W(s)$ are frequency dependent parameters of the source. $B_{1}(\theta)$ is thought of as a reflection coefficient which represents the contribution to the total electric field above the earth from the sub-surface layers. For a twolayered conductor $B_{3}(\theta)=0$ so that $E_{x}^{3}$ will not become infinite with depth. The other $A_{n}(\theta)$ and $B_{n}(\theta)$ values can be obtained since the tangential components of $E_{x}^{n}$ and $H_{y}^{n}$ must be continuous across layer boundaries. This leads to

$$
B_{1}(\theta)=\frac{R-1}{R+1}
$$

where

$$
R=\frac{s(Q+1)}{\theta_{2}(Q-1)}
$$

and

$$
Q=\frac{\theta_{2}+\theta_{3}}{\theta_{2}-\theta_{3}} \cdot \exp \left(2 \theta_{2} d_{1}\right)
$$

where $d_{1}$ is the thickness of the first conducting layer.

This can, of course, be extended to more layers, but we will be concerned only with a two-layered earth. 
The surface magnetic field values obtained by taking the curl of (7) are expressed as:

$$
\left.H_{y}^{1}(y)=-\frac{i}{\omega \mu_{0}} \int_{0}^{\infty} s\left\{1-B_{1}(\theta)\right\} V(s) \cos (s y)+W(s) \sin (s y)\right\} \mathrm{d} s
$$

and

$$
\left.H_{z}^{1}(y)=\frac{i}{\omega \mu_{0}} \int_{0}^{\infty} s\left\{1+B_{1}(\theta)\right\} V(s) \sin (s y)-W(s) \cos (s y)\right\} \mathrm{d} s .
$$

The aperiodic solution is obtained for the magnetic field values by writing

and

$$
H_{y}(y, t)=\int_{-\infty}^{\infty} H_{y}^{1}(y, \omega) \cdot F(\omega) \exp (i \omega t) \mathrm{d} \omega,
$$

$$
H_{z}(y, t)=\int_{-\infty}^{\infty} H_{z}^{1}(y, \omega) \cdot F(\omega) \exp (i \omega t) \mathrm{d} \omega,
$$

where $F(\omega)$ is the Fourier transform of the time variation $f(t)$ of the current source.

$$
F(\omega)=\frac{1}{2 \pi} \int_{-\infty}^{\infty} f(t) \exp (-i \omega t) \mathrm{d} t
$$

$H_{y}(y, t)$ and $H_{z}(y, t)$ are the magnetic field responses as a function of time.

4. The Aperiodic Solution for the Symmetric and Non-Symmetric Source

The two magnetic field components of both sources were calculated along the surface of a horizontally layered earth with two layers. The upper layer was of thickness $50 \mathrm{~km}$ and resistivity $100 \mathrm{ohm}-\mathrm{m}$. The second layer was of infinite depth and resistivity $10 \mathrm{ohm}-\mathrm{m}$.

The symmetric source has a current distribution of

$$
I=I_{0} \mathrm{e}^{-y^{2} / 2 \kappa^{2}},
$$

where the half-width $(\kappa)$ is $240 \mathrm{~km}$. The non-symmetric source has a current distribution of

$$
I=I_{0}(y-b) \mathrm{e}^{-a(y-b)} \cdot u(y-b)
$$

where $b=700 \mathrm{~km}, a=2.1 \times 10^{-4} \mathrm{~km}^{-1}$ and $u$ is the unit step function. (Refer to Fig. 2 for relative source positions.)

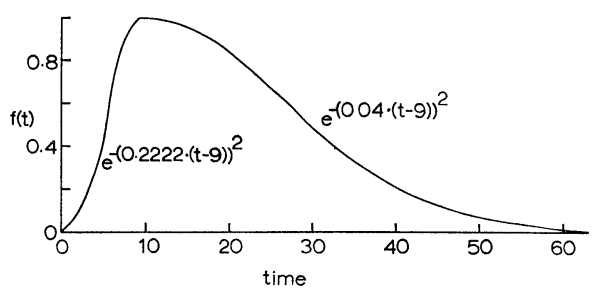

Fig. 3. Time variation of the current sources. (Time scale in minutes) 

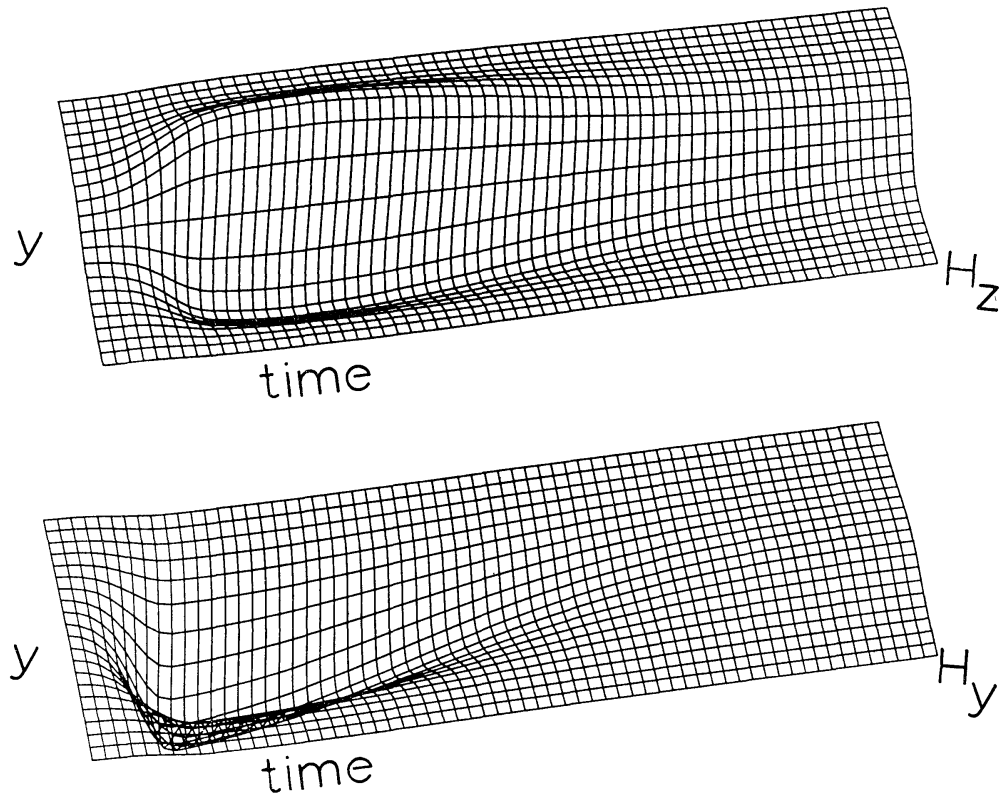

Fig. 4. Varration of $H_{z}$ (above) and $H_{y}$ (below) with time for the symmetric source.
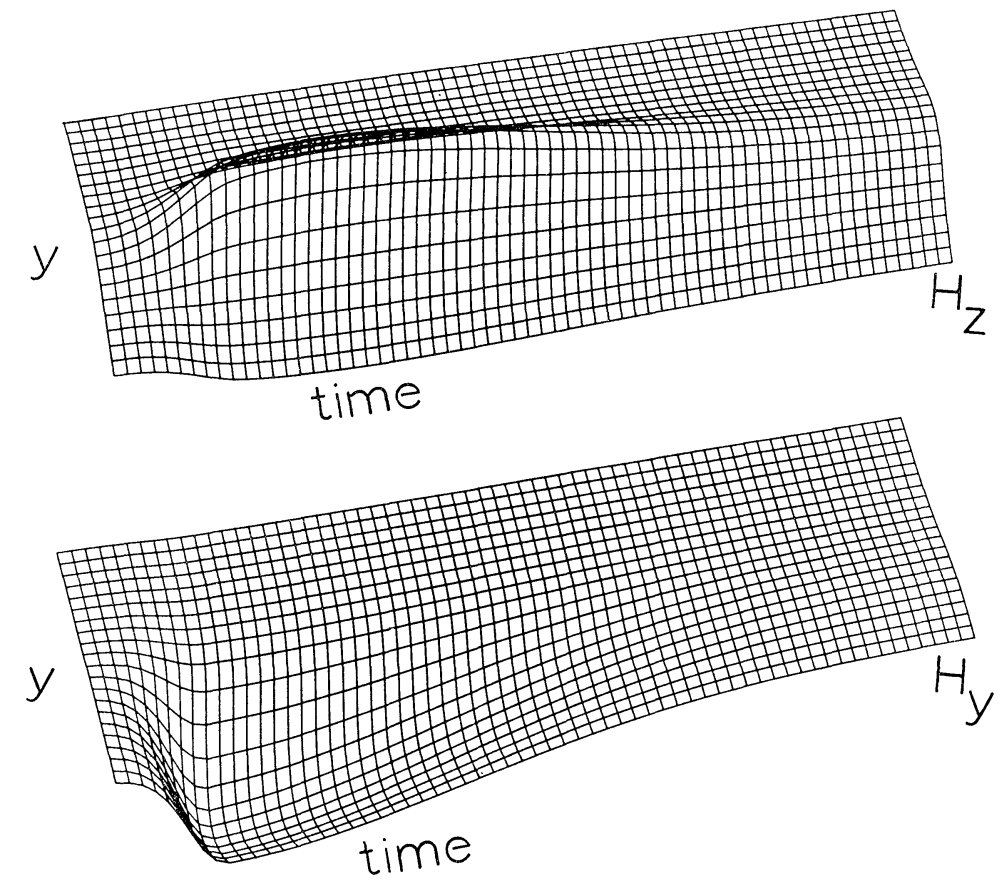

Fig. 5. Variation of $H_{z}$ (above) and $H_{y}$ (below) with time for the non-symmetric source. 
The time variation of both current distributions was the same;

$$
f(t)=\mathrm{e}^{-d^{2}(t-9)^{2}} .
$$

For $0<t \leqq 9$ minutes $d=0.2222$ and for $9<t \leqq 63$ minutes $d=0.040$. The time variation is shown in Fig. 3.

The Fourier spectrum of the current distribution time variation was band limited to less than $1 \mathrm{~Hz}$ since we have assumed that displacement currents may be neglected. The numerically calculated frequency spectrum of the time function chosen is negligible above $0.01 \mathrm{~Hz}$ and was obtained through the use of the FFT program.

The time variations of the magnetic field components $H_{y}$ and $H_{z}$ over the region of interest on the surface are shown in Fig. 4 for the symmetric source and Fig. 5 for the non-symmetric source. Starting with time $t=0$ a total time span of 63 minutes is shown at time intervals of one minute.

\section{The Aperiodic Spatially-Varying Double Current System}

As outlined before, the aperiodic spatially varying source is obtained by the superposition of the symmetric and non-symmetric field component solutions.

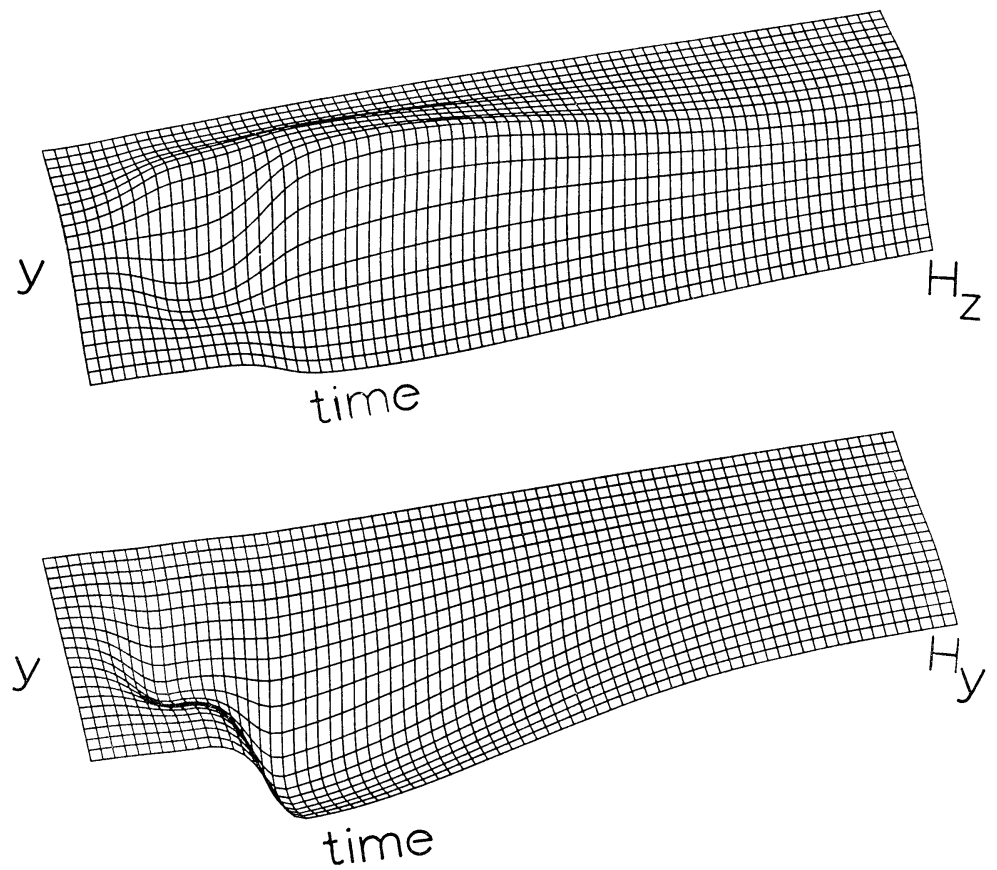

Fig. 6. Variation of $H_{z}$ (above) and $H_{y}$ (below) with time for the superimposed symmetric and non-symmetric source with the non-symmetric lag 10 minutes in time behind the symmetric source. 

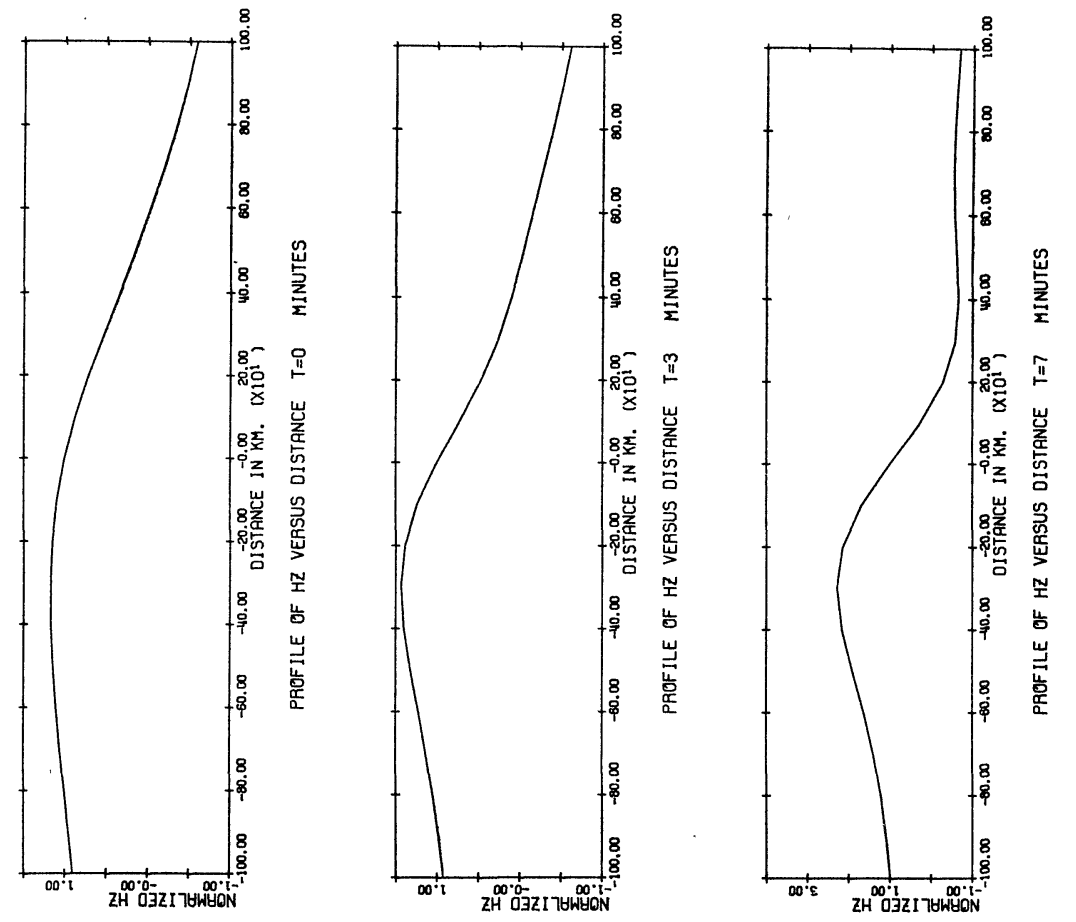

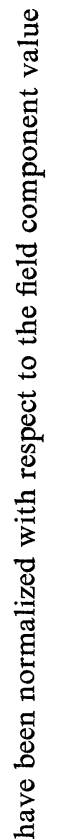
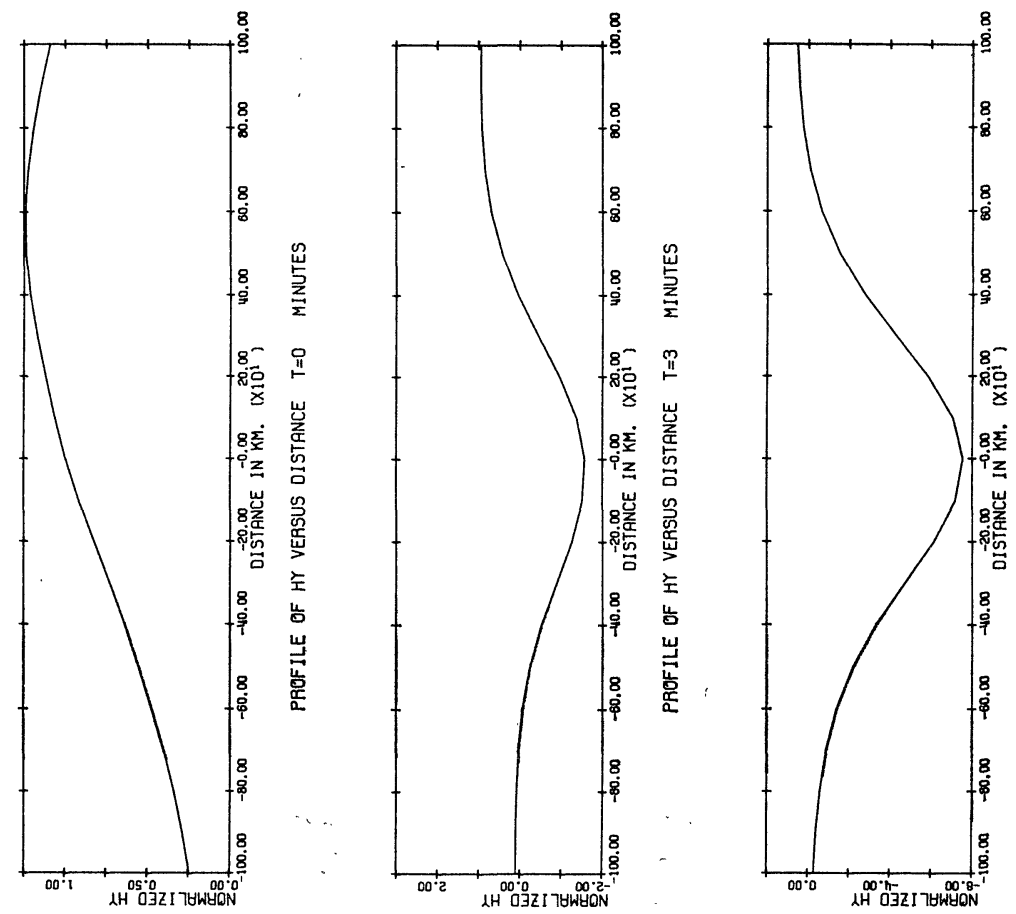

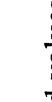

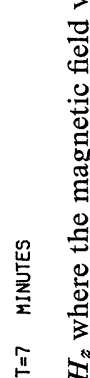

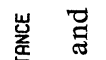

点

के

एक

台苛范

荘 焉

产

की

$\underset{\infty}{\infty}$ 

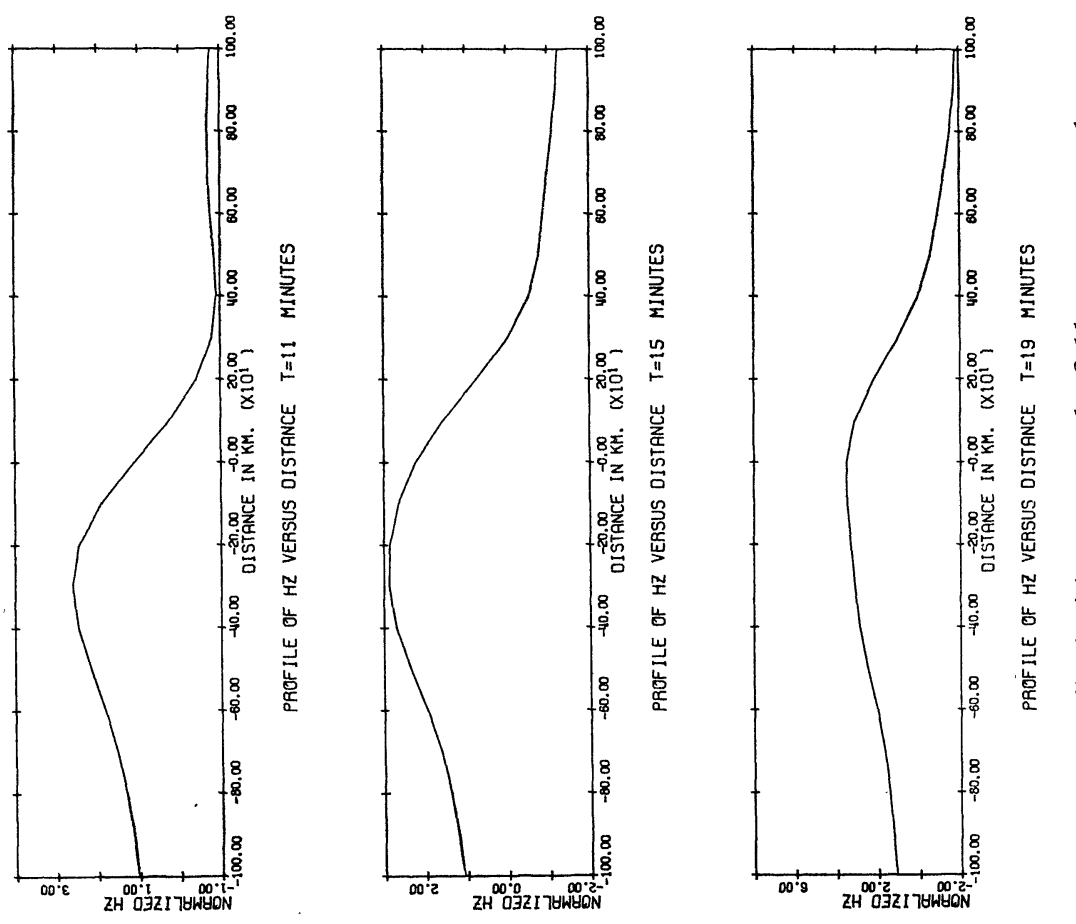

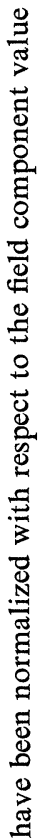
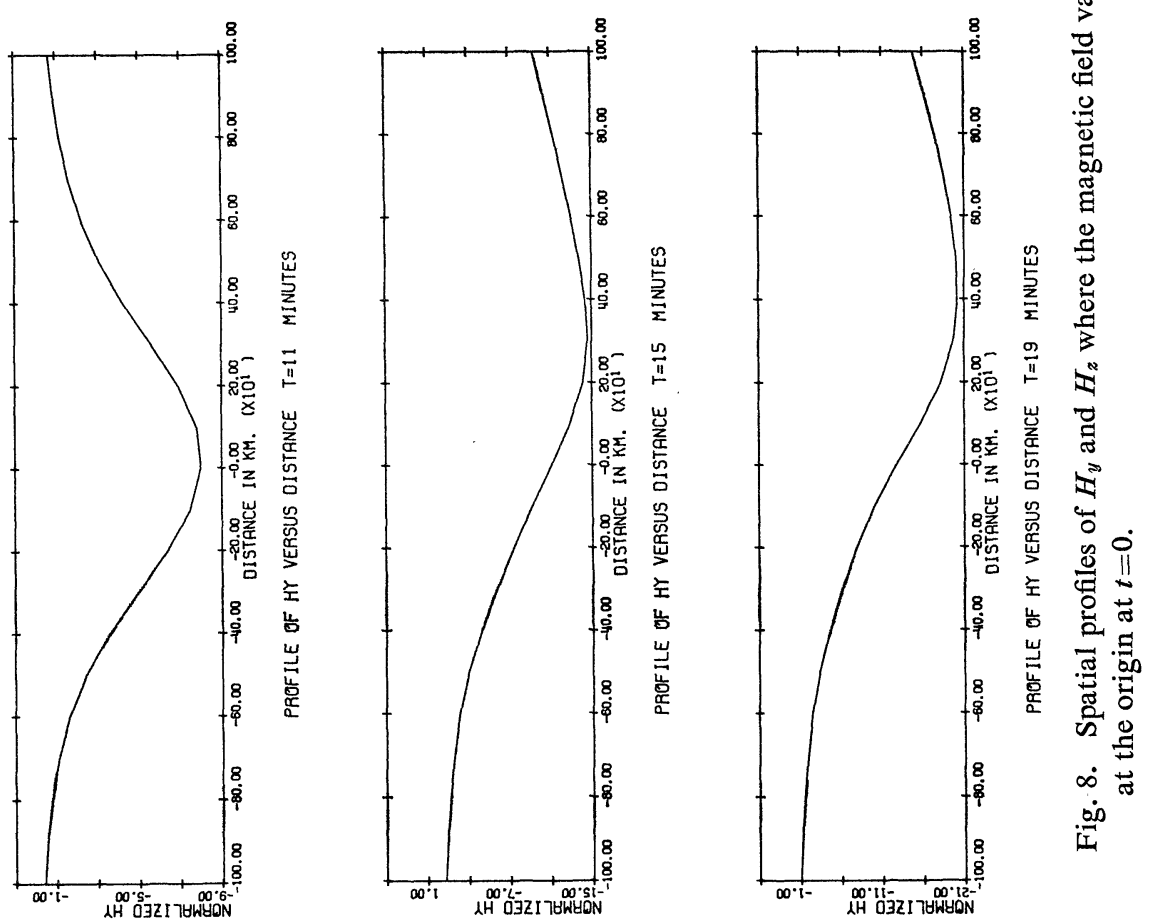

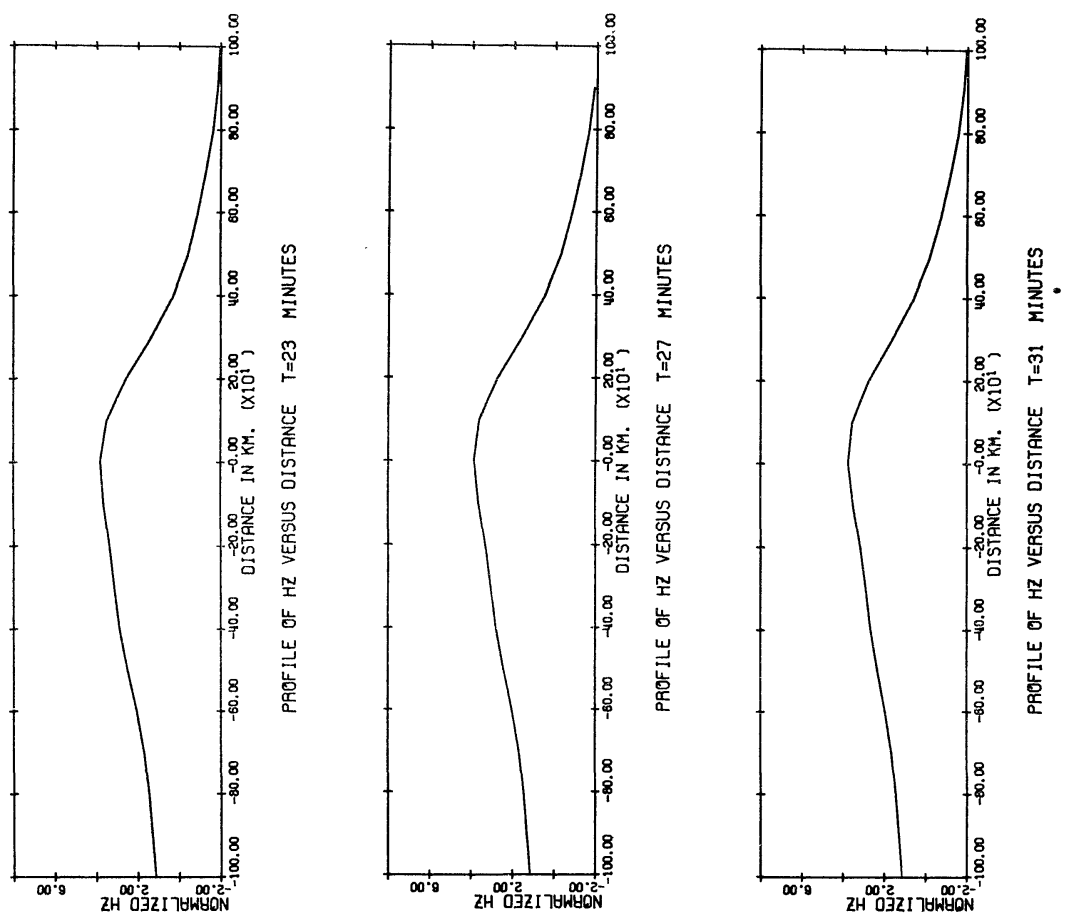

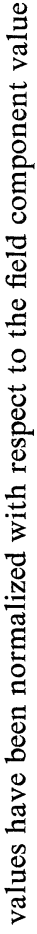
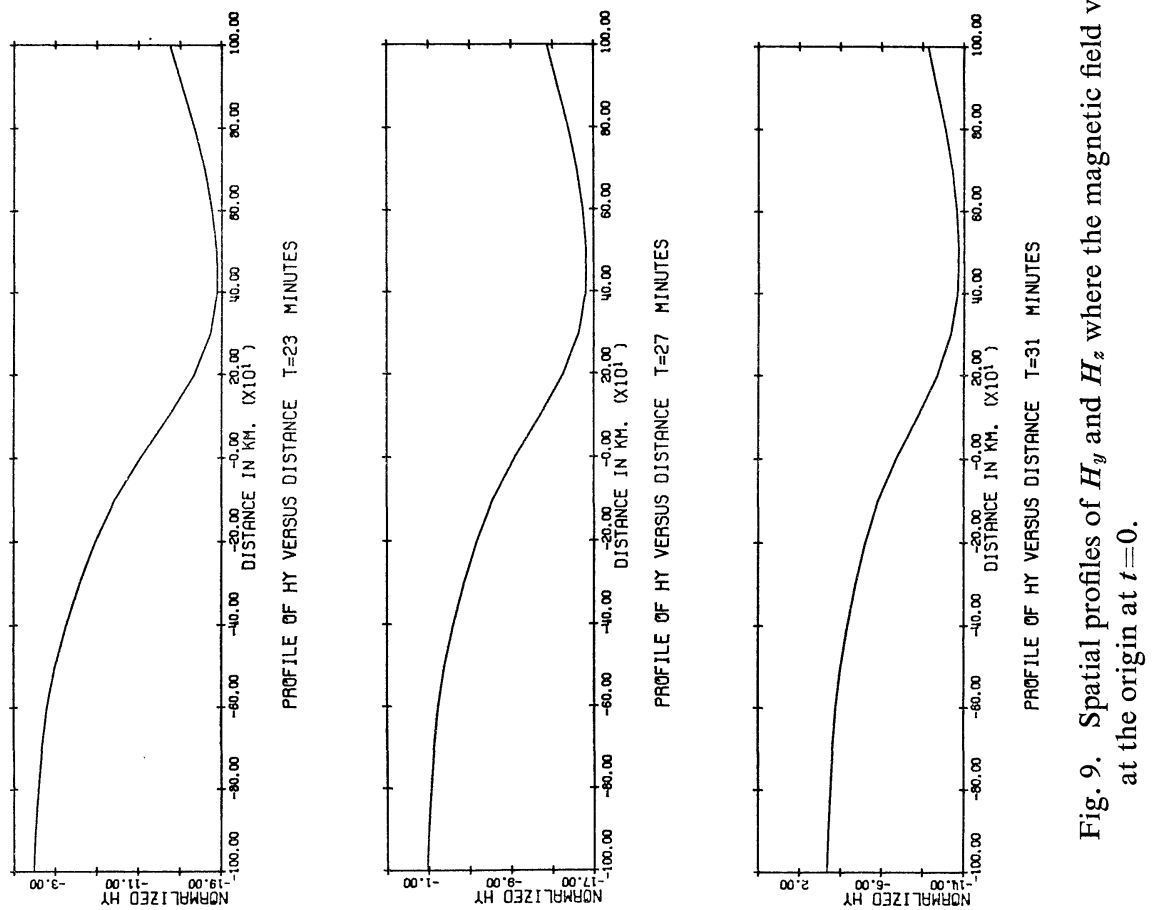

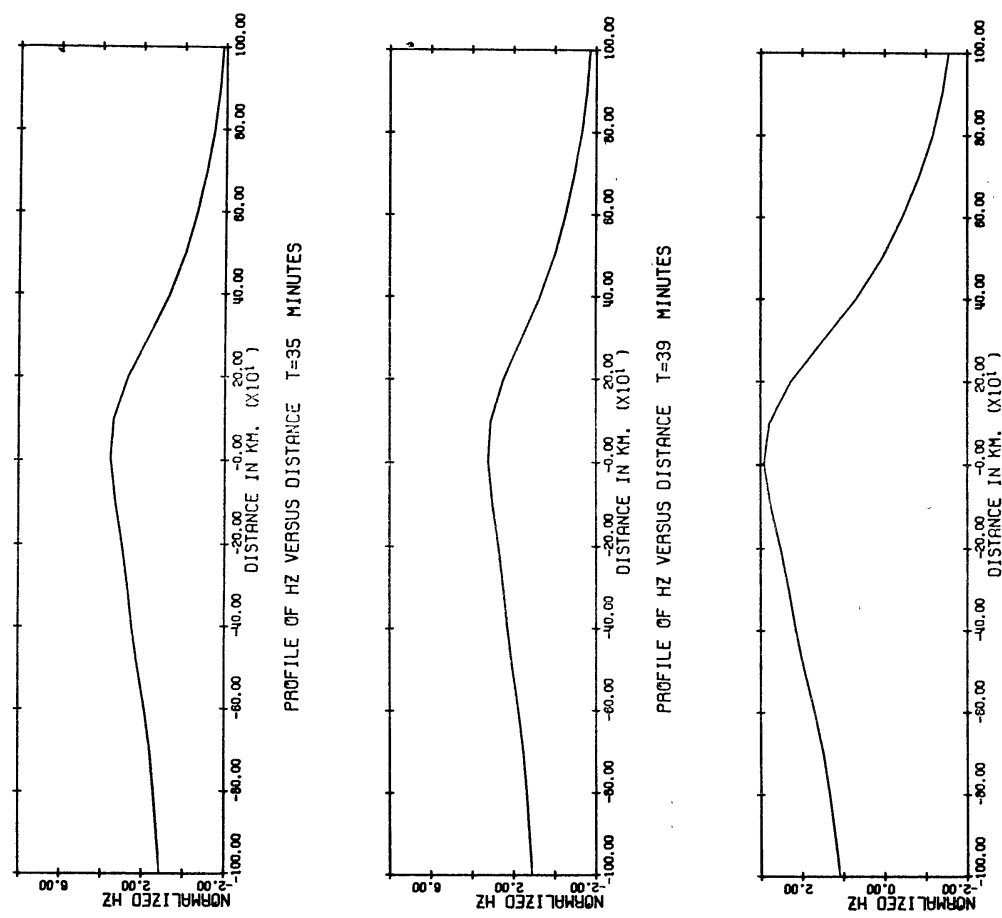

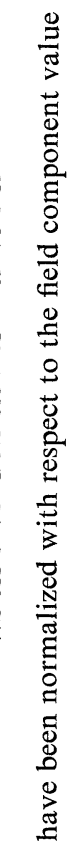
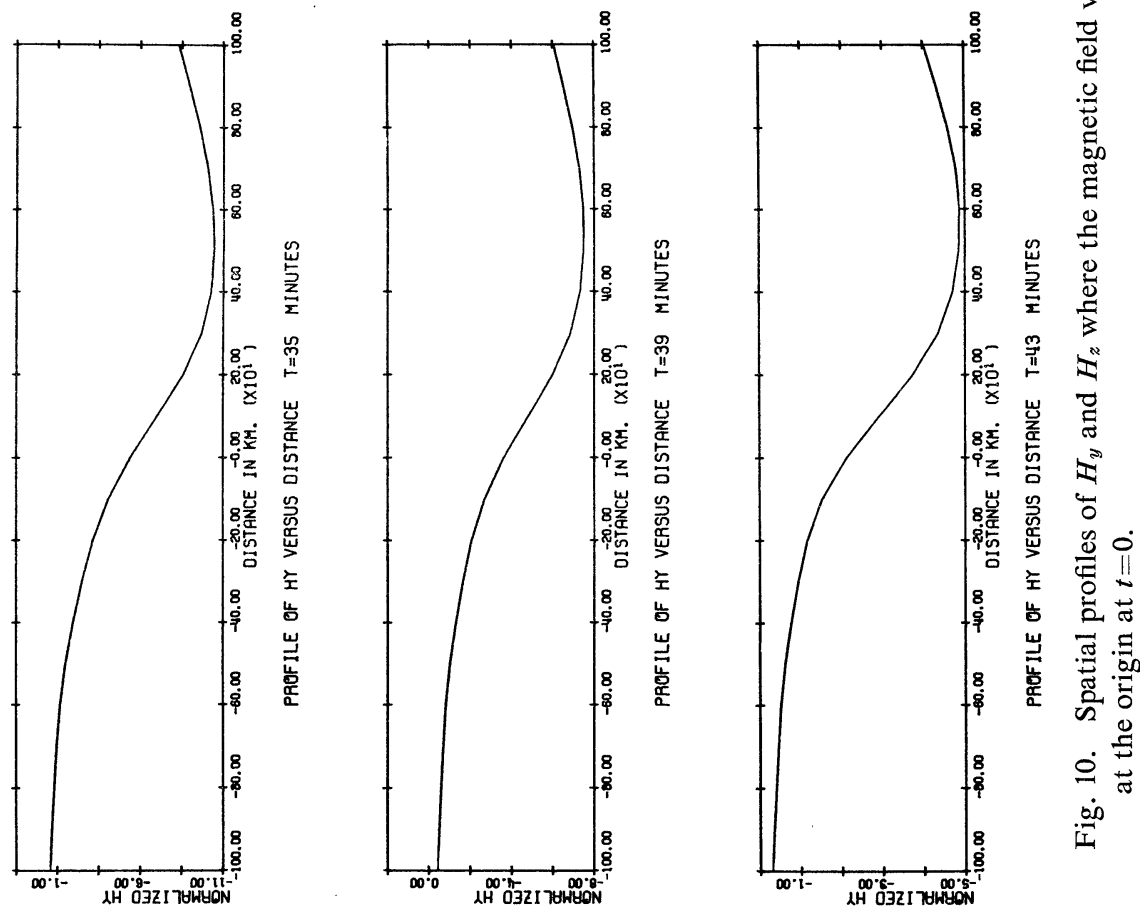

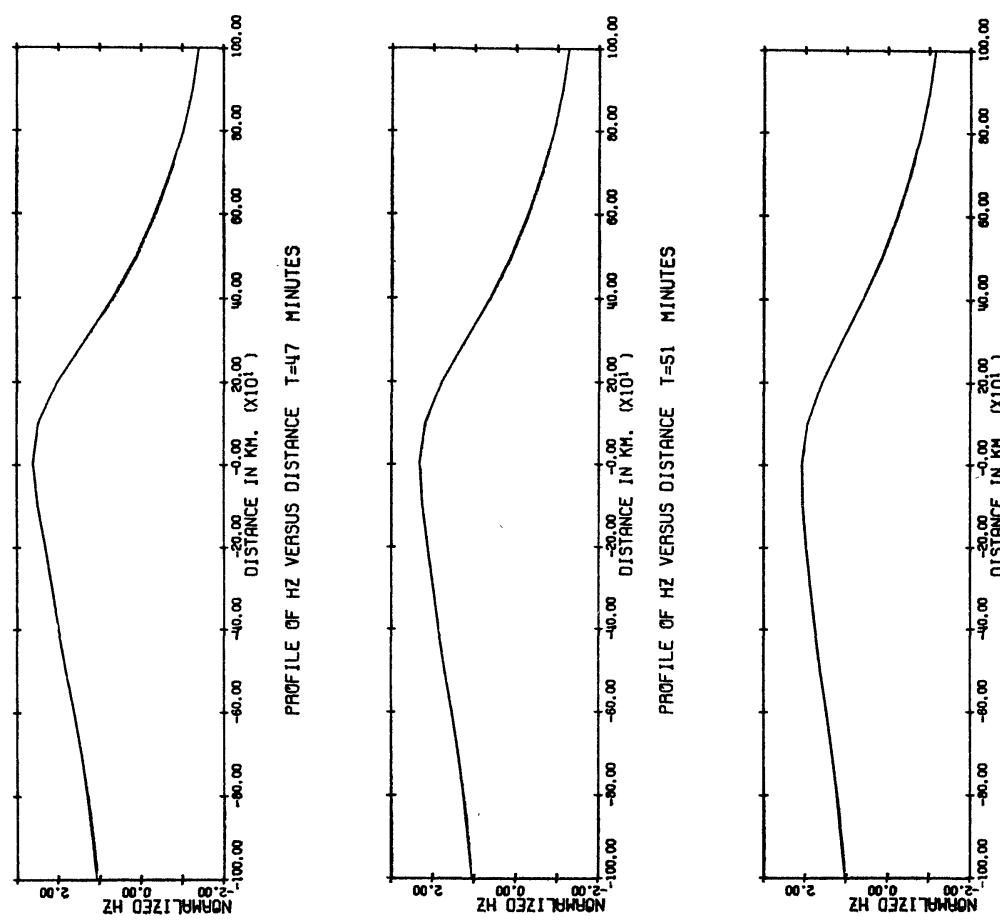

焉
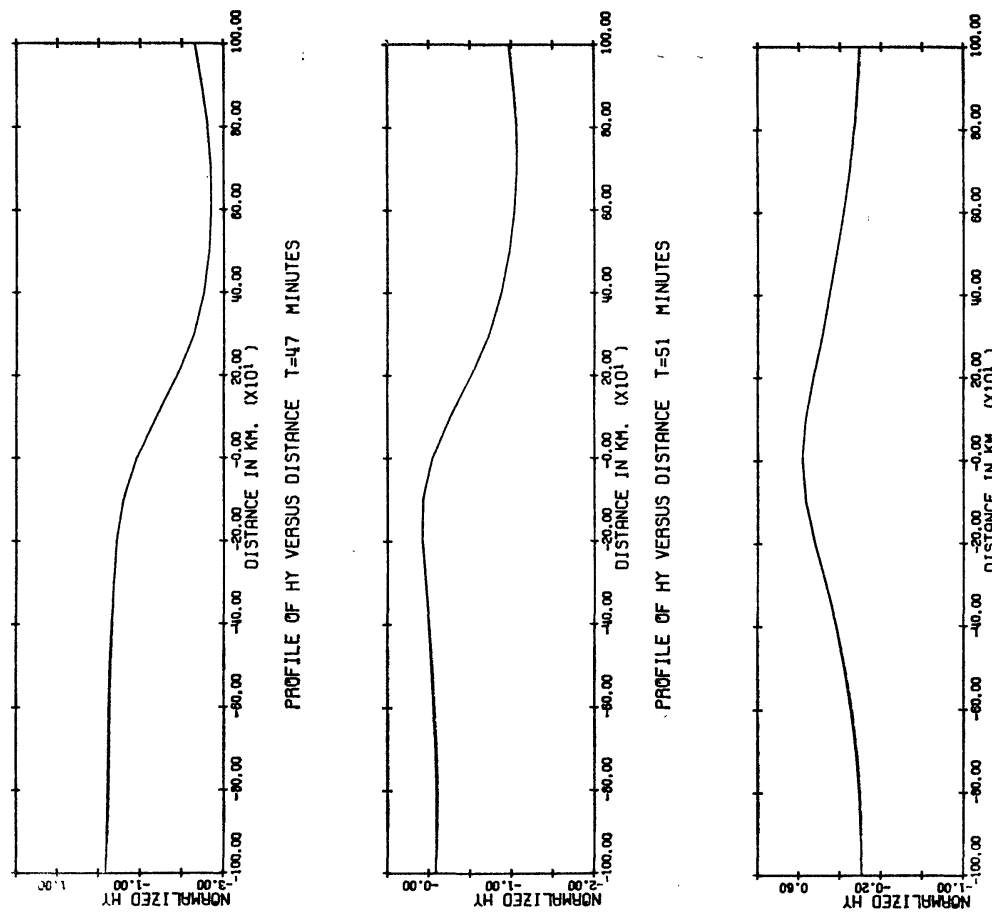
Electromagnetic Induction in the Earth by an Aperiodic Non-Uniform Current Source 51
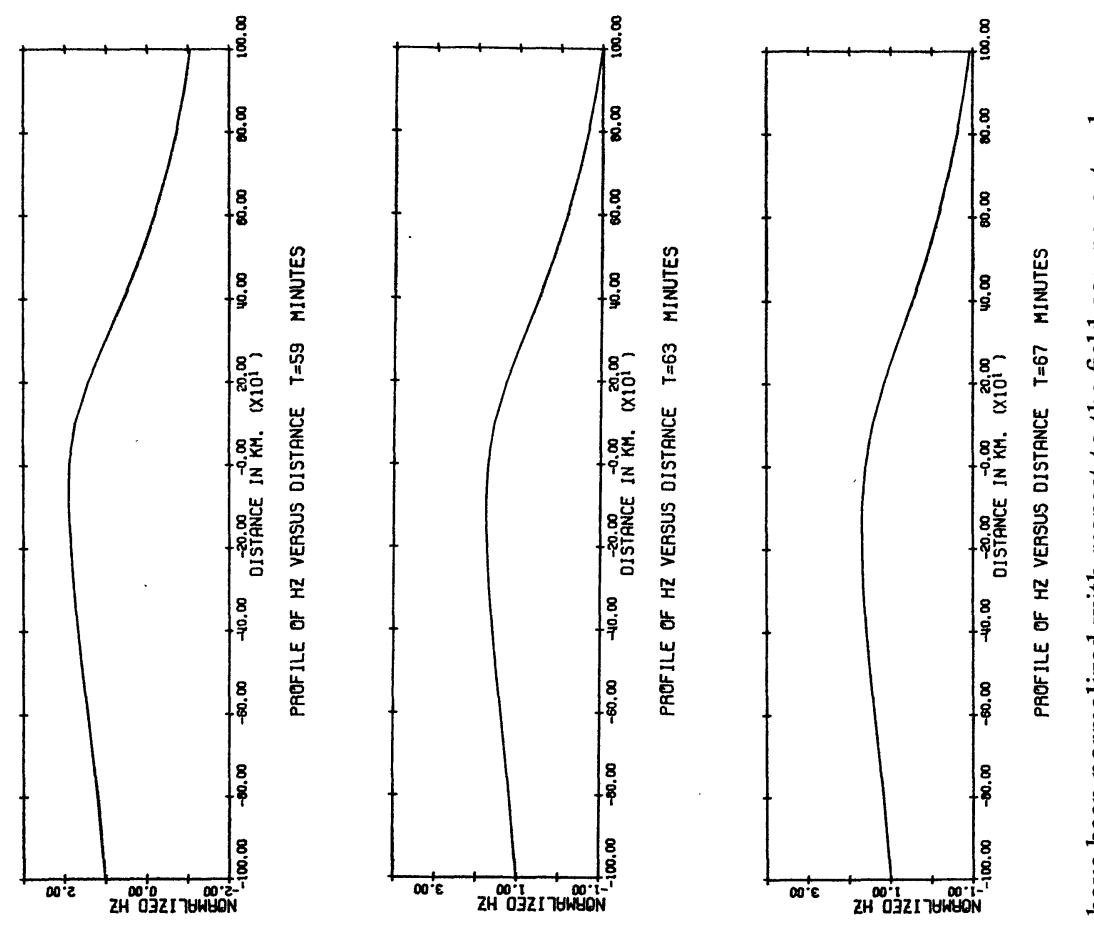

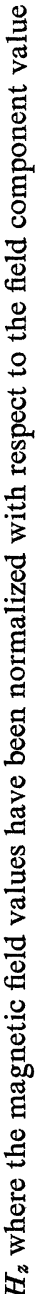
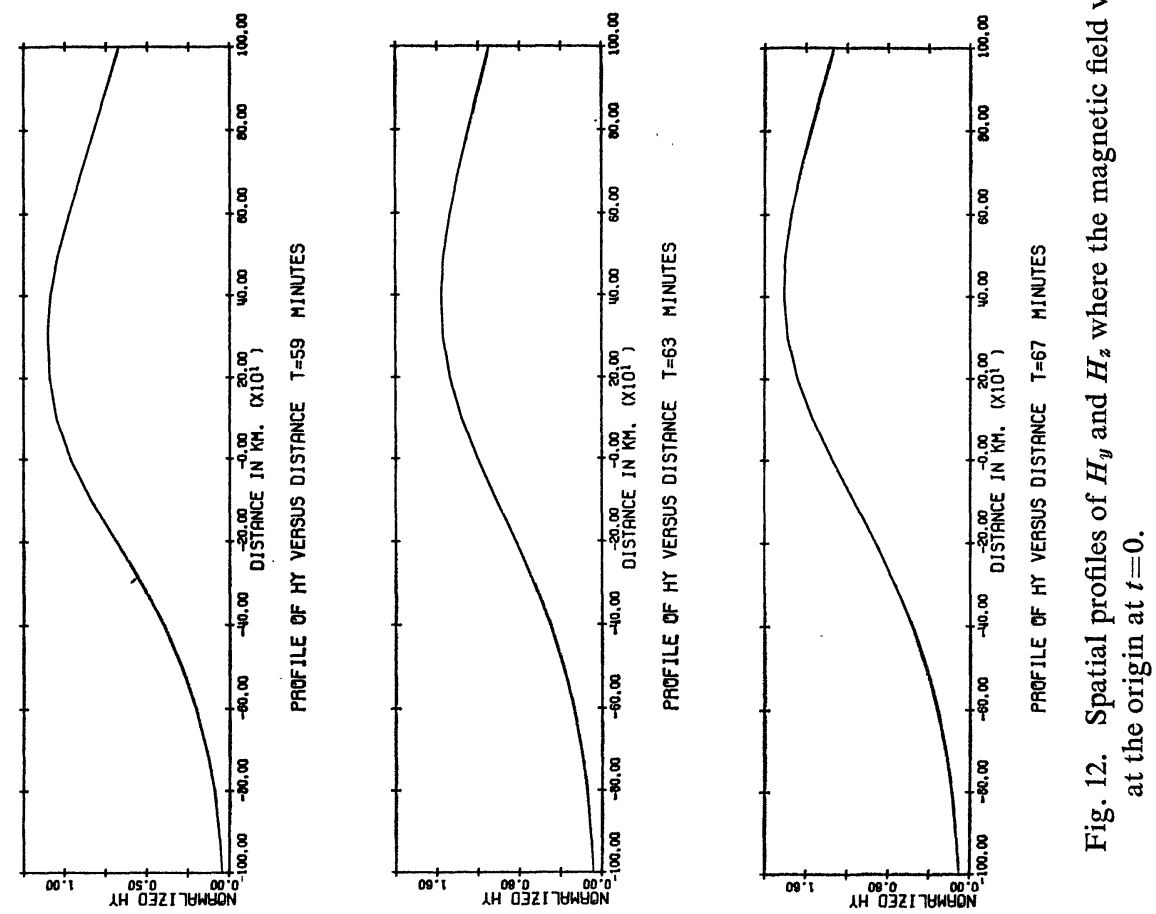
The non-symmetric source lags the symmetric source by ten minutes in time. The time variations for the magnetic components at the surface due to the composite source are shown in Fig. 6 with a time span of 74 minutes and intervals the same as in Figs. 4 and 5.

\section{Discussion}

The symmetric source, which is centered with respect to the origin produces an $H_{y}$ magnetic field component at the surface which is also symmetric about the origin. The $H_{z}$ cross-over point (the point at which $H_{z}$ equals zero) coincides with the origin as can be seen in Fig. 4. The non-symmetric source, having most of its current concentration positioned to the right of the region where the calculations of the magnetic field components were obtained, shows a high degree of non-symmetry in both the $H_{y}$ and $H_{z}$ magnetic field components as is evidenced in Fig. 5. The fact that the current concentration is to the right of the region of interest is clearly indicated by the position of the maximum value of $H_{y}$ and the cross-over point of $H_{z}$. When the two sources are superimposed the magnetic field component configuration "moves" spatially with time. $H_{y}$ exhibits two maxima in time at different positions along the surface as can be seen in Fig. 6. The cross-over point of $H_{z}$ shifts to the right with time as can best be seen in Figs. 7, 8 and 9, which give spatial profiles of the $H_{y}$ and $H_{z}$ components for intervals of time four minutes apart starting at $t=0$.

The non-symmetry in the surface profile of the $H_{y}$ component (Fig. 7) for the first two times is due to the fact that the contribution of the non-symmetric source was held constant for the first 10 minutes. The initial values of $H_{y}$ at $t=0$ of Fig. 5 were used for the non-symmetric source contribution of Fig. 6 for $t=0$ minutes through $t=10$ minutes. In Fig. 6 for $t>63$ minutes, the symmetric source was held constant at its final value ( $t=63$ minutes, Fig. 4$)$. The same procedure was followed for the calculation of the $H_{z}$ component in Figs. $6,7,8$ and 9 .

Kisabeth and Rostoker (1971) and Kisabeth (1972) have observed a latitudinal movement of the $H_{z}$ cross-over associated with polar magnetic substorms, and the work discribed here also indicates this movement. It is apparent that the work described here supports the observations and interpretations by Kisabeth and Rostoker (1971) and Kisabeth (1972) with reference to the existence and behavior of double current systems.

This work was supported by the National Reserch Council of Canada.

\section{REFERENCES}

Hermance, J.F. and W.R. Peltier, Magnetotelluric fields of a line current, J. Geophys. Res., 75, 3351-3356, 1970. 
HibBs, R.D. and F.W. Jones, Electromagnetic induction in the earth by a symmetric nonuniform source, Geophys. J. R. astr. Soc., 32, 269-277, 1973 a.

HibBs, R.D. and F.W. Jones, Electromagnetic induction in the earth by a non-symmetric nonuniform source, J. Geomag. Geoelectr., 25, 75-86, 1973 b.

Jones, F.W. and A.T. PrICE, The perturbations of alternating geomagnetic fields by conductivity anomalies, Geophys. J. R. astr. Soc., 20, 317-334, 1970.

Kisabeth, J.L. and G. Rostoker, Development of the polar electrojet during polar magnetic substorms, J. Geophys. Res., 76, 6815-6828, 1971.

Kisabeth, J.L., The dynamical development of the polar electrojets, Ph. D. thesis, University of Alberta, 1972.

Peltier, W.R. and J.F. Hermance, Magnetotelluric fields of a Gaussian electrojet, Can. J. Earth Sci., 8, 338-346, 1971.

Price, A.T., Electromagnetic induction in a semi-infinite conductor with a plane boundary, Quart. J. Mech. Appl. Math., 3, 385-410, 1950.

WAIT, J.R., On the relation between telluric currents and the earth's magnetic field, Geophys., 19, 281-289, 1954. 